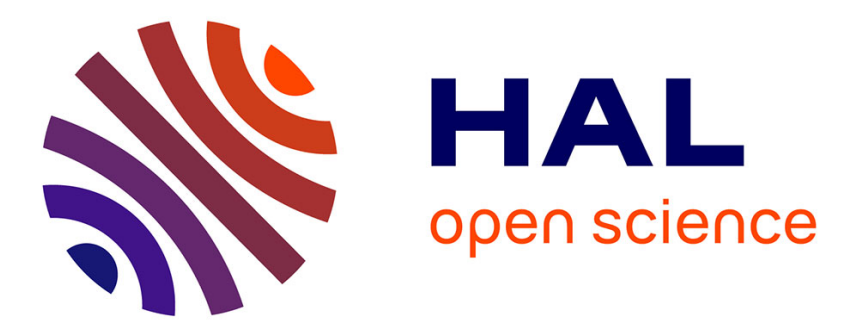

\title{
Largeur des raies de résonance magnétique de l'état 6 3P1 du mercure et diffusion multiple des photons 2537 $\AA$
}

\author{
Jean-Pierre Barrat
}

\section{- To cite this version:}

Jean-Pierre Barrat. Largeur des raies de résonance magnétique de l'état $63 \mathrm{P} 1 \mathrm{du}$ mercure et diffusion multiple des photons 2537 A. J. Phys. Radium, 1958, 19 (11), pp.858-862. 10.1051/jphysrad:019580019011085800 . jpa-00235955

\section{HAL Id: jpa-00235955 https://hal.science/jpa-00235955}

Submitted on 1 Jan 1958

HAL is a multi-disciplinary open access archive for the deposit and dissemination of scientific research documents, whether they are published or not. The documents may come from teaching and research institutions in France or abroad, or from public or private research centers.
L'archive ouverte pluridisciplinaire HAL, est destinée au dépôt et à la diffusion de documents scientifiques de niveau recherche, publiés ou non, émanant des établissements d'enseignement et de recherche français ou étrangers, des laboratoires publics ou privés. 


\title{
LARGEUR DES RAIES DE RÉSONANCE MAGNÉTIQUE DE L'ÉTAT $6{ }^{3} \mathrm{P}_{1}$ DU MERCURE ET DIFFUSION MULTIPLE DES PHOTONS $2537 \AA$
}

\author{
Par J. P. BARRAT, \\ Laboratoire de Physique, E. N. S., Paris.
}

\begin{abstract}
Résumé. - Les mesures par diverses méthodes (résonance magnétique ou dépolarisation magnétique) de la durée de vie $\tau$ du niveau $6{ }^{3} P_{1}$ du mercure conduisent en réalité à une " durée de cohérence " $T$ plus longue que $\tau$, tendant vers $\tau$ aux très basses pressions de vapeur. Ce phénomène est dû à la diffusion multiple des photons de résonance optique par les atomes de mercure. Celle-ci se produit de façon indépendante pour chaque composante hyperfine de la raie $2537 \AA$; elle est responsable également de la dépolarisation de la raie de résonance optique quand la pression de vapeur augmente. La théorie permet de prévoir des relations entre la durée de cohérence et le taux de polarisation pour une composante hyperfine d'un isotope déterminé, ou entre les durées de cohérence relative aux différentes composantes hyperfines. Elle donne aussi la forme théorique des raies de résonance magnétique dans le cas où on isole les photons diffusés deux fois par les atomes de mercure. Les résultats expérimentaux sont en accord satisfaisant avec les prévisions théoriques.
\end{abstract}

\begin{abstract}
Several methods used to measure the lifetime $\tau$ of the $6{ }^{3} P_{1}$ state of mercury (double resonance and Hanle's method), lead, in fact, to a "coherence time " $T$, longer than $\tau$, and equal to $\tau$ at very low vapour pressures.

This effect is due to imprisonement of 2537 optical resonance radiation. It happens in a completely independent way for the different hyperfine components of the 2537 line. The same effect is responsible for the depolarisation of the 2537 line at high vapour pressures.

The theory gives relations between $\mathrm{T}$ and the degree of polarisation of a given hyperfine component; and the relations between the T's of the different isotopes. It gives also the theoretical shape of the magnetic resonance lines when optical resonance photons have been scattered only twice. Experimental results are in good agreement with theory.
\end{abstract}

I. Introduction. - La méthode de la " double résonance " de Brossel-Kastler [1] a été appliquée par Brossel à l'étude du premier niveau excité $6^{3} P_{1}$ des différents isotopes du mercure [2]. Les formes de raie observées étaient en excellent accord avec les prévisions théoriques. En particulier, l'extrapolation en champ de radiofréquence nul de la largeur des raies permet de déterminer un paramètre $T$ que Brossel avait interprété comme étant la durée de vie $\tau$ du niveau. Toutefois, Blamont, reprenant à l'occasion de ses recherches sur l'effet Stark les mesures de Brossel [3], constata que $T$ est fonction des conditions expérimentales, en particulier de la pression de vapeur du mercure dans la cellule à résonance. Pour des conditions géométriques déterminées, $T$ est fonction croissante du nombre $N$ d'atomes de mercure par $\mathrm{cm}^{3}$. Les raies s'affinent quand la pression de vapeur augmente. Cependant, dans tous les cas la forme de la raie reste en accord avec la formule théorique dans laquelle intervient la grandeur variable $T$ que nous appellerons "durée de cohérence ". De nombreux arguments ont permis d'attribuer ce phénomène à la diffusion multiple des photons par les atomes de la vapeur de mercure [4]: aux pressions de vapeur élevées, les photons émis à l'intérieur de la cellule ont une probabilité non négligeable d'être réabsorbés dans la cellule même; ils subissent ainsi plusieurs diffusions avant de parvenir à l'observateur.
II. Interprétation qualitative des effets observés. - A. EFFETS DÉJA CONNUS DE LA DIFFUSION MULTIPLE. - 1) Si l'on coupe brusquement le rayonnement incident, l'intensité de la lumière de résonance optique décroît avec une constante de temps $\tau^{\prime}$, égale à $\tau$ aux très basses pressions de vapeur, supérieure à $\tau$ quand la diffusion multiple intervient. Des mesures de $\tau^{\prime}$ en fonction de la pression de vapeur ont été effectuées par Garrett [5] qui détermina $\tau$ par cette méthode, et plus récemment par Holstein [6].

2) Le taux de polarisation $P$ de la lumière de résonance optique décroît quand la pression de vapeur des atomes diffusants augmente. Ce phénomène, attribué souvent à des collisions, trouve en réalité son origine dans la diffusion multiple, au moins dans le cas du mercure [7].

B. Caractère des effets de la diffusion MULTIPLE. - 1) La structure hyperfine de la raie de résonance du mercure $2537 \AA\left(6^{1} S_{0}-6^{3} P_{1}\right)$ est grande devant la largeur Doppler des composantes. Il est donc possible d'exciter sélectivement certains isotopes pairs et certaines composantes hyperfines des isotopes impairs dans un mélange isotopique (par exemple, en éclairant du mercure naturel par une lampe contenant l'isotope ${ }^{202} \mathrm{Hg}$ pratiquement pur), et un transfert d'excitation par diffusion multiple des photons doit se faire indépendamment pour chaque composante de la raie. 
Les effets de la diffusion multiple, dans des conditions géométriques déterminées, ne dépendent que du nombre $N$ des atomes de l'isotope diffusant par $\mathrm{cm}^{3}$, et non de la concentration des autres isotopes. Cette conclusion a été vérifiée pour les grandeurs $T$ et $P$ (pour les isotopes ${ }^{202} \mathrm{Hg}$ et ${ }^{198} \mathrm{Hg}$ dans $\mathrm{Hg}$ naturel, et pour l'isotope ${ }^{202} \mathrm{Hg}$ pur) [4, $8,9]$. Aux basses pressions de vapeur la diffusion multiple n'intervient plus et $T$ tend vers une limite, la même pour tous les isotopes, qui est,

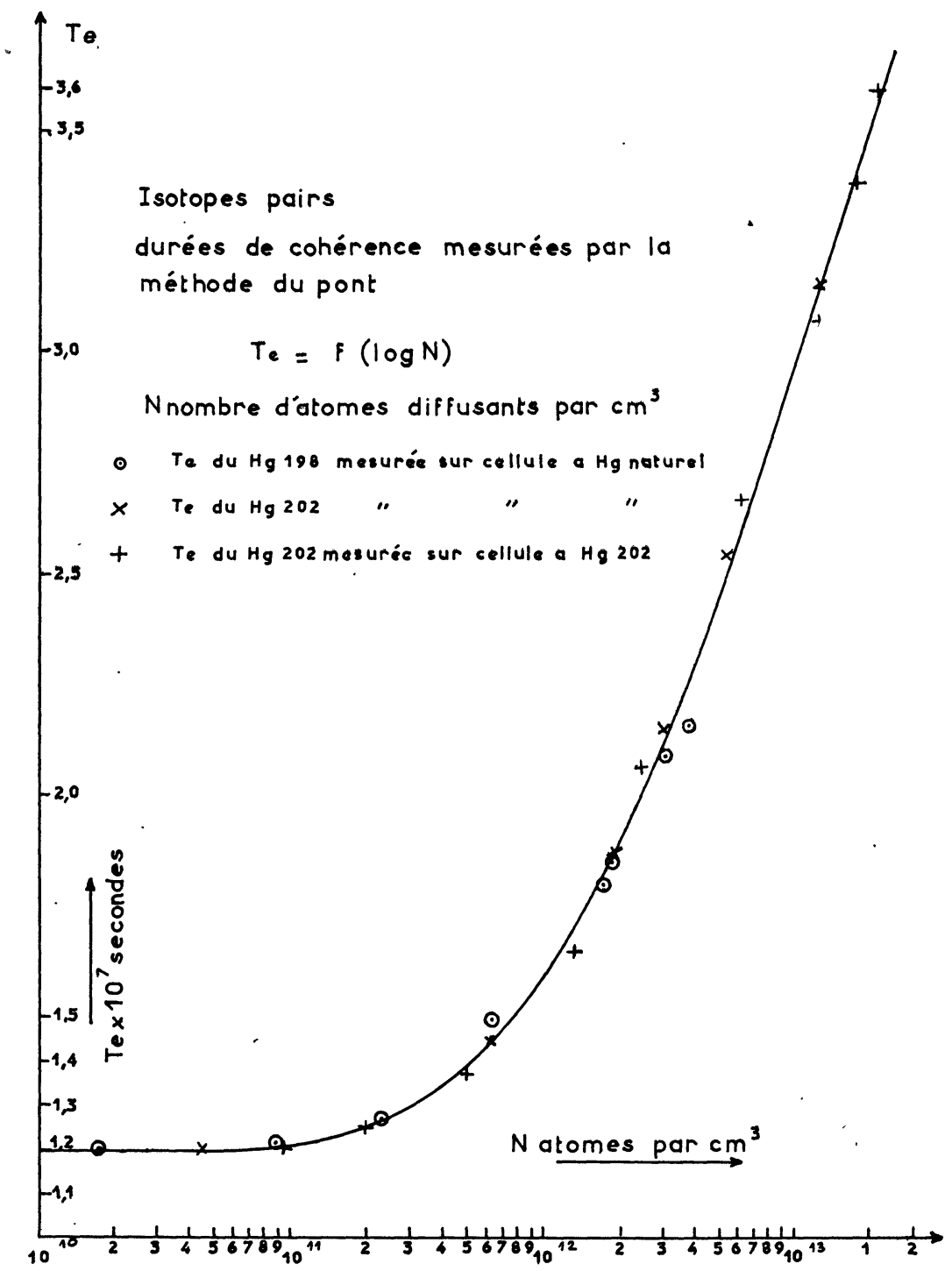

FIG. 1.

sauf phénomène imprévu, la durée de vie vraie $\tau$ de l'atome isolé ( $\left.\tau=1,18.10^{-7} \mathrm{sec}\right)$ (fig. 1).

2) L'effet dû à des collisions interatomiques ne dépendrait que de la fréquence de celles-ci, c'est-àdire de la température et de la pression de. vapeur, il resterait inaffecté par un changement des conditions géométriques. Il n'en est pas de même d'un phénomène lié à la diffusion multiple $[4,7,8]$. C'est d'ailleurs à cet effet qu'est due la découverte du phénomène.
C. Caractère cohérent de la diffusion mulTIPLE. - La diffusion multiple serait incohérente si chaque atome émettait des intensités lumineuses des diverses polarisations $\sigma_{+}, \sigma_{-}$et $\pi$, correspondant aux transitions $\Delta m=+1,-1,0$ totalement incohérentes, ne dépendant que des probabilités de présence dans les sous-niveaux Zeeman de l'état excité. Les équations permettant d'étudier la diffusion multiple ne feraient intervenir que les populations des sous-niveaux. On peut montrer [4] 


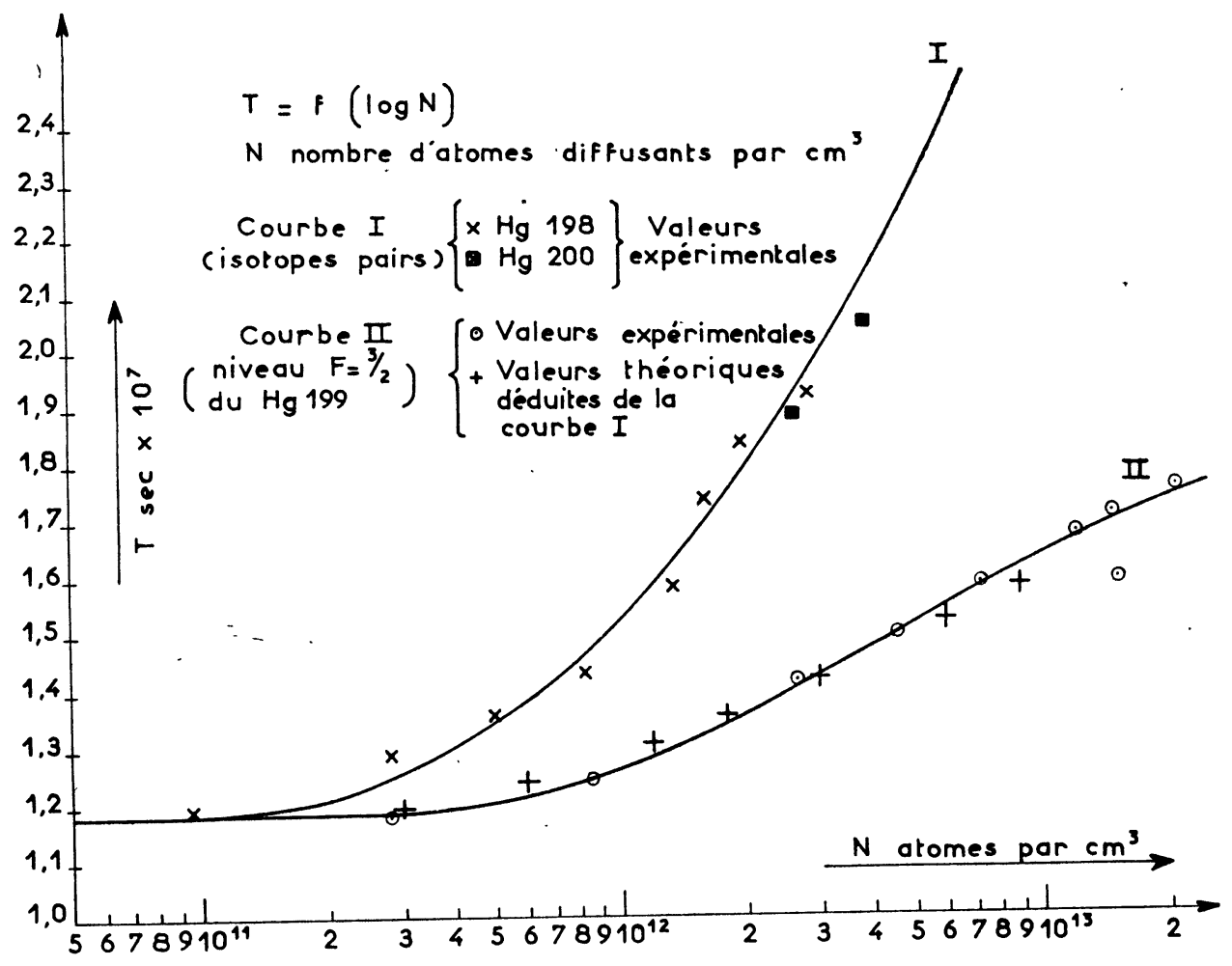

FIG. 2.

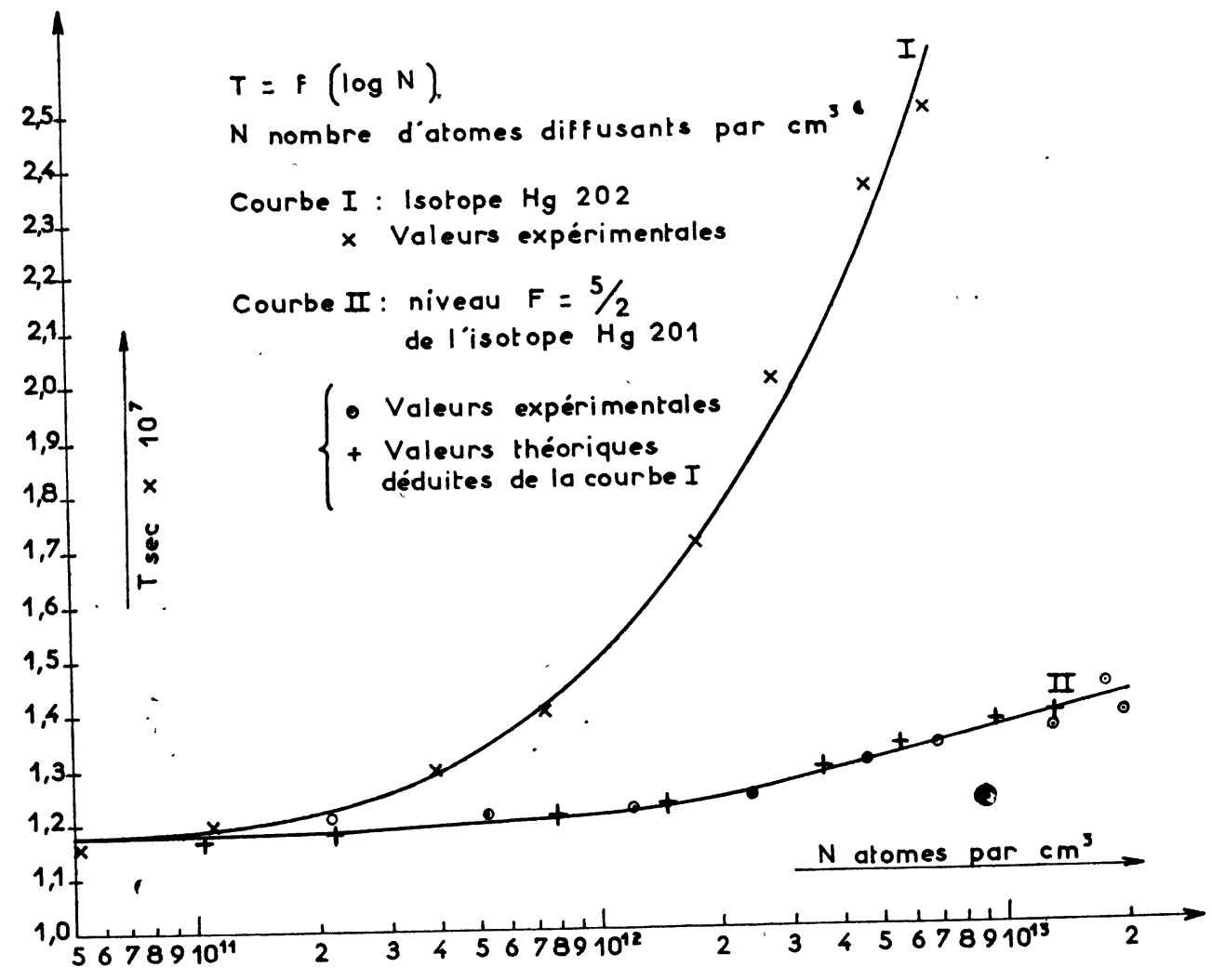

FIg. 3. 
qu'un tel processus permet d'interpréter l'augmentation de $\tau^{\prime}$ et la diminution de $P$ quand $N$ augmente ; mais une telle conception conduit au contraire à prévoir un élargissement des raies de résonance magnétique.

Il faut donctenir compte en détail de la cohérence dans la diffusion multiple : la fonction d'onde d'un atome $I$ dans la vapeur a la forme $\Psi^{I}(t)=\sum_{i} C_{i}^{I}(t) \Psi_{i}^{I}$, où $\Psi_{i}^{I}$ est la fonction d'onde qui représente le niveau i de cet atome, les $C_{i}^{I}(t)$ étant des coefficients. Non seulement les $\left|C_{i}^{I}(t)\right|^{2}$ (probabilités de présence) des divers atomes sont reliés entre eux, mais aussi les $C_{\boldsymbol{i}}^{\boldsymbol{I}}(t)$ eux-mêmes. Leur évolution dans le temps est régie par l'équation de Schrödinger, tenant compte de la théorie quantique du rayonnement.

III. Résultats quantitatifs. - Le calcul par cette méthode des effets de la diffusion multiple peut se faire dans le cas de chaque niveau hyperfin $F$ d'un isotope du mercure de spin nucléaire $I$, en champ magnétique assez faible pour ne pas découpler $\vec{I}$ et $\vec{J}$. Il conduit à la définition d'un paramètre $x$, représentant la probabilité moyenne pour qu'un photon qui vient d'être émis par un atome soit réabsorbé au moins une fois avant de sortir de la cellule. La définition même de $x$ résulte de l'approximation selon laquelle cette probabilité est indépendante de la position de l'atome émetteur. $x$ est une fonction bien déterminée de $F, I$ et $N$ dans des conditions géométriques fixées : $x=f\left[\frac{(2 F+1) N}{3(2 I+1)}\right]$. Son calcul explicite est impraticable et on doit se concenter d'un calcul approché, en assez bon accord (à $10 \%$ près environ) avec les déterminations expérimentales.

Selon les résultats théoriques, toutes les grandeurs mesurables, telles que $T, \tau^{\prime}, P$, sont des fonctions bien déterminées, en général simples

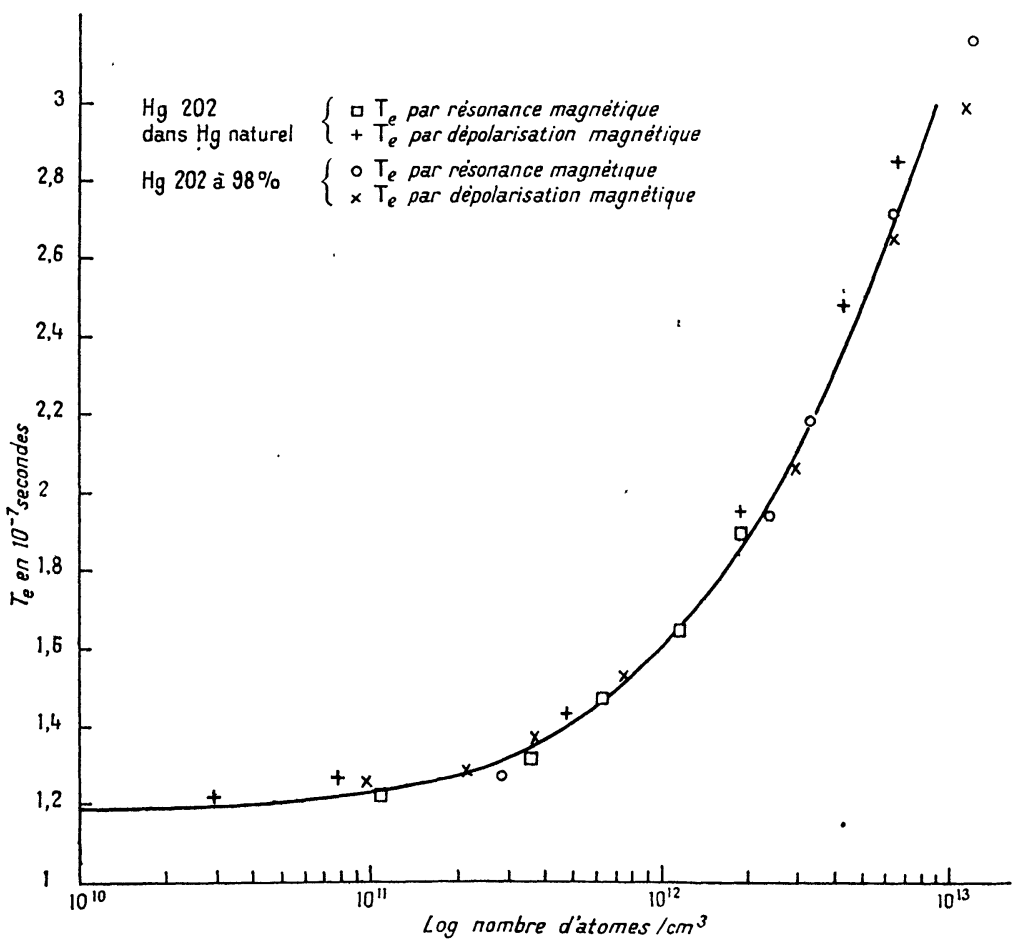

Fig. 4.

de $x$, pour $I$ et $F$ donnés. La vérification de ces conclusions peut se faire, soit en comparant directement les valeurs de $x$ obtenues par l'intermédiaire de $T$ ou $P$ aux valeurs théoriques en fonction de $N$ (accord à $10 \%$ près environ). soit en éliminant $N$ entre 2 formules donnant 2 grandeurs telles que $T_{I, F}$ et $P_{I, F}$ ou $T_{I, F}$ et $T_{I^{\prime}, F^{\prime}}$, et en comparant la relation théorique et la relation expérimentale entre ces grandeurs.

L'accord est alors, en général, bon à quelques pour cent près. En particulier, ont été étudiées de cette manière :

- la relation entre $T_{I=0, F=1}$ ct $P_{I=0, F=1}$ (isotopes pairs) $[4,8]$;

- la relation entre $T_{I=1 / 2, F=3 / 2}\left(\right.$ isotope ${ }^{199} \mathrm{Hg}$ ) et $T_{I=0, F=1}($ fig. 2) $[8,10]$;

- la relation entre $T_{I=3 / 2, F=5 / 2}, T_{I=3 / 2, F=3 / 2}$ (isotope ${ }^{201} \mathrm{Hg}$ ) et $T_{I=0, F=1}[10]($ fig. 3$)$;

- la relation entre $T_{I=1 / 2, F=3 / 2}$ et $P_{I=1 / 2}$ (en excitation " broad line ») [8]; 
- la relation entre $T_{I-0, F-1}$ et la grandeur $T_{I-0, F-1}^{\prime}$ que l'on obtient lorsque l'on essaie de mesurer la durée de vie par la méthode de la dépolarisation magnétique [11, 9] (fig. 4).

En outre le calcul permet de prévoir les résultats obtenus si l'on isole des photons ayant été diffusés un nombre de fois déterminé, 2 fois par exemple. L'expérience a pu être réalisée [12] et les résultats sont en accord avec les prévisions, tant en ce qui concerne la largeur des raies de résonance magnétique que leur forme.

\section{BIBLIOGRAPHIE}

[1] Brossel (J.) et Kastler (A.), C. R. Acad. Sc., 1949, $229,1213$.

[2] Brossel (J.), Ann. Physique, 1952, 7, 622. Brossel (J.), et Bitter (F.), Phys. Rev., 1952, 86, 308.

[3] Blamont (J.), Ann. Physique, 1957, 2, 551.

[4] Guiochon (M. A.), Blamont (J.) et Brossel (J.), J. Physique Rad., 1957, 18, 99 ; C. R. Acad. Sc., 1956, 243, 1859. Guichon (M. A.), Diplôme d'Études Supérieures, Paris, 1956.

[5] Garrett (P. H.), Phys. Rev., 1932, 40, 779.

[6] Holstein (T.), Phys. Rev., 1947, 72, 1212. Holstein (T.), Alpert (D.) et McCoubrey (A. O.), Phys. Rev., 1949, 76, 1257 ; Phys. Rev., 1951, 82, 567; Phys. Rev., 1952, 85, 985.

[7] Rollet (N.), Brossei (J.) et Kastler (A.), $C . R$. Acad. Sc., 1956, 242, 240.

[8] Boutron (F.), Diplôme d'Études Supérieures, Paris, 1957.

[9] Barrat (J. P.), C. R. Acad. Sc., 1957, 244, 2785.

[10] Boutron (F.), Barrat (J. P.) et Brossel (J.), C. $R$. Acad. Sc., 1957, 245, 2250.

[11] Voir Mitchell et Zemansky, Resonance Radiation and Excited Atoms, p. 262.

[12] Barrat (J. P.) et Brossel (J.), C. R. Acad. Sc., 1958, 246, 2744.

\section{DISCUSSION}

G. J. Gorter. - "Si j'évalue bien les ordres de grandeur, le libre parcours moyen des photons dans la diffusion multiple est considérable. Est-ce exact ?"

J. P. Barrat. - "Au centre de la raie d'absorption Doppler, le libre parcours moyen des photons est $2.10^{12} / N \mathrm{~cm}$, où $N$ est le nombre d'atomes diffusant par $\mathrm{cm}^{3}$. Pour un isotope pur à $0^{\circ} \mathrm{C}$, $N \simeq 6.10^{12}$, ce qui donne un libre parcours moyen de $3 \mathrm{~mm}$ environ. ")

C. J. Gorter. - "Pourrait-on en modulant le libre parcours moyen et en étudiant le halo de j'image de la source lumineuse, obtenir des données nouvelles sur les résonances et, la diffusion multiple?"

J. P. Barrat. - "Pour un photon de fréquence déterminée, le coefficient d'absorption, dans nos expériences où la séparation Zeeman des niveaux est petite devant l'effet Doppler (champs magnétiques inférieurs à 100 œrsteds), est déterminé par la forme Doppler de la raie. On ne peut le moduler en modulant le champ magnétique. Par contre, une telle modulation serait possible en faisant varier le champ magnétique d'une valeur nulle à une valeur de plus de 1000 œrsteds, correspondant à un effet Zeeman nettement supérieur à l'effet Doppler. "

E. E. Schneider. - " Il me semble que dans la diffusion multiple des photons, l'assemblage des atomes se comporte classiquement en ce qui concerne l'effet de résonance. On pourrait se demander s'il y a une analogie avec le comportement classique d'une assemblée de noyaux dans la résonance nucléaire.»

J. P. Barrat. - "Dans le cas de la résonance magnétique nućléaire, la mise en phase provient du fait que le champ H. F. a la même phase en tous les points où se trouvent les noyaux, étant donné que la longueur d'onde de l'onde H. F. est grande devant les distances atomiques. Au contraire, la longueur d'onde de l'onde optique $(\lambda=2537 \AA)$ est petite devant les distances interatomiques. La différence de phase de l'onde est déterminée pour 2 atomes déterminés, mais elle varie aléatoirement d'un couples d'atomes à un autre. Je comparerais plutôt l'ensemble des atomes à un ensemble d'antennes classiques. Les équations déterminant ces phénomènes sont très comparables. ")

A. Kastler. - " La théorie développée par J. P. Barrat a peut-être des applications au cas des excitons, étudié par M. Balkanski. ”

M. Balkanski. - "En effet, comme M. Kastler l'a remarqué, la théorie développée par J. P. Barrat semble pouvoir être d'un très grand secours dans le domaine de la Physique des Solides lorsqu'il s'agit de traiter la propagation des excitons dans les semi-conducteurs. L'analogie phénoménologique est complète et l'on peut redéfinir le problème de l'exciton en se basant sur les caractéristique de la diffusion multiple, à condition d'introduire, è dehors des photons transversaux habituels, les photons longitudinaux et les phonons. ” 